\title{
Perceived and real tic suppression ability and its relation to impulsivity
}

Valerie C. Brandt, Ph.D. ${ }^{1}$, Katja Herrmann, M.Sc. ${ }^{2}$, Julia Kerner auch Koerner, Ph.D. ${ }^{3,4}$, Alexander Münchau, Prof., M.D. ${ }^{2}$

${ }^{1}$ Department of Psychology, Centre for Innovation in Mental Health, University of Southampton, UK

${ }^{2}$ Department of Paediatric and Adult Movement Disorders and Neuropsychiatry, Center for Brain, Behavior and Metabolism, University of Lübeck, Lübeck, Germany

${ }^{3}$ Helmut-Schmidt University, Hamburg, Germany

${ }^{4}$ Center for Research on Individual Development and Adaptive Education of Children at Risk (IDeA), Frankfurt, Germany

\section{Corresponding author:}

Valerie Brandt

Department of Psychology, Centre for Innovation in Mental Health, Building 44, University of Southampton, SO17 1BJ

Southampton,

UK

Phone: 00442380591820

Email: V.C.Brandt@,soton.ac.uk

\section{Word count: 570}

Running title: How impulsivity relates to tic suppression ability

Key words: Habit reversal training; exposure with response prevention; tic suppression; Tourette syndrome; ADHD

Disclosures: This study was not funded by a funding body, none of the authors have any conflicts of interest to disclose regarding their funding or their work on this study. 
The question has been raised whether Gilles de la Tourette syndrome (GTS) patients with comorbid Attention Deficit Hyperactivity Disorder (ADHD) may benefit less from behavioral therapy, i.e., habit reversal training and exposure with response prevention because their ticsuppression ability is limited ${ }^{1}$. However, a recent study did not find ADHD to moderate treatment response ${ }^{2}$. Findings regarding the association between ADHD symptoms and tic suppression ability have been mixed: Studies using measures of subjectively perceived ability to suppress tics found a relation to $\mathrm{ADHD}^{3}$, whereas studies using objective measures did not ${ }^{4}$. However, no study has compared both in one sample. We hypothesized that ADHD symptoms will affect GTS patients' subjectively perceived ability to suppress tics but not their objective ability.

Our study addresses tic suppression ability in GTS patients in relation to comorbid ADHD. Thirty-five patients (mean age 31.60 +/- 12.07 SD; 9 female) with a DSM-5 GTS diagnosis participated and gave informed written consent prior to the study that was approved by the local ethics committee. Three-minute videos of face and upper body in two conditions (free ticcing, tic suppression - no reinforcement) were scrutinized for tics by two independent ticraters (reliability: intraclass correlation coefficients $>.99, p<.001$ ). Mean tic scores were calculated across raters (free: $64 \pm 38.52$; suppression: $50 \pm 30.74$ ). An inhibition potency score was calculated [(sum tics free ticcing - sum suppression) / sum tics free ticcing $\left.)^{5}\right]$. Subjective tic suppression ability was assessed with item 10 of the Premonitory Urge for Tics Scale, ADHD with the German ADHD self-rating scale (ADHD-SB) ${ }^{6}$. Correlations reported are Spearman's rho (see table 1).

Patients who scored higher on the ADHD measure, particularly impulsivity, thought that they were less able to suppress their tics successfully but correlations with inhibition potency showed that there was no association between ADHD symptoms and objective tic inhibition ability (table 1, supplementary figure 1). A non-significant correlation between self-reported 
ability to suppress tics and inhibition potency $(r=.32, p=.07)$ suggested that patients were only moderately good at judging their own tic inhibition ability.

Taken together, ADHD symptoms in adult GTS patients, particularly self-perceived impulsivity, affect patients' perception or judgment of how well they are able to suppress tics, while their actual ability to suppress tics for a short period of time appears to be nearly unaffected by ADHD symptoms. This study provides first insights into the discrepancy regarding tic suppression ability and ADHD symptoms in the literature ${ }^{3,4}$. The results might depend on the method used to assess tic suppression.

ADHD patients have difficulties judging their own performance ${ }^{7}$. Our findings suggest that GTS patients who perceive themselves to be highly impulsive are self-inattentive or overcritical judging their own performance, underestimating their ability to suppress tics. This notwithstanding, recent evidence shows that a co-occurring ADHD diagnosis does not moderate treatment response to behavioral interventions, while positive participant expectancy does ${ }^{2}$. Future studies might investigate whether developing a more realistic selfperception with those patients who perceive themselves to be highly impulsive could have a positive effect on therapeutic outcome.

There are several limitations to the study. The sample size is small, hence, the results should be replicated in a large sample. Furthermore, the videos of tic suppression were only $3 \mathrm{mins}$ long, instead of a commonly used and more reliable 10mins. Long-term tic suppression might involve different processes, e.g. sustained attention or the ability to delay gratification. Moreover, we did not control for depression and anxiety. 
Table 1. Clinical data of $n=35$ patients with Tourette syndrome

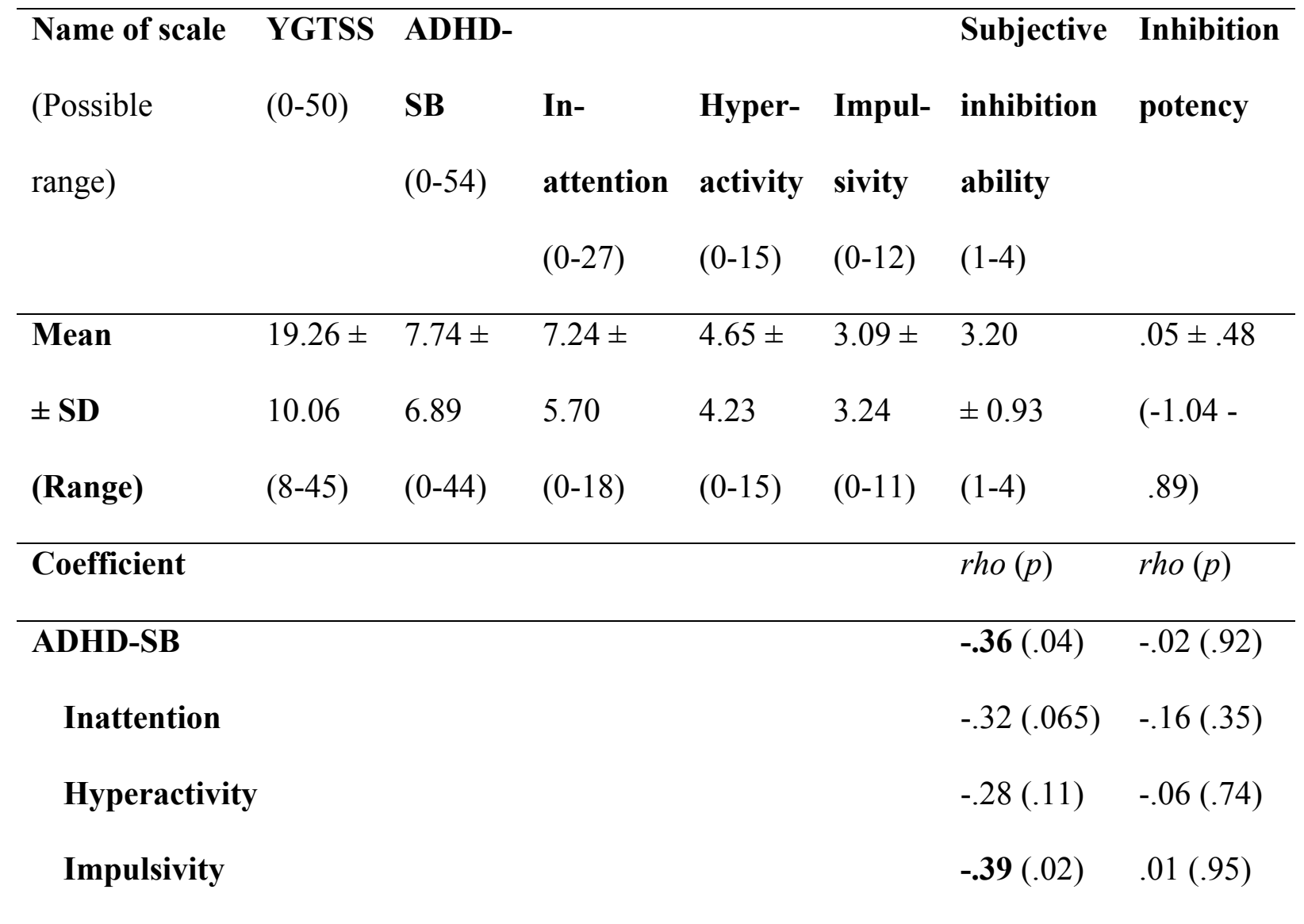

YGTSS = Yale Global Tic Severity Scale; ADHD-SB = ADHD self-rating scale, subjective tic suppression ability was assessed with item 10 of the Premonitory Urge for Tic Scale. According to the ADHD-SB, 10 patients fulfilled criteria for an ADHD diagnosis. 


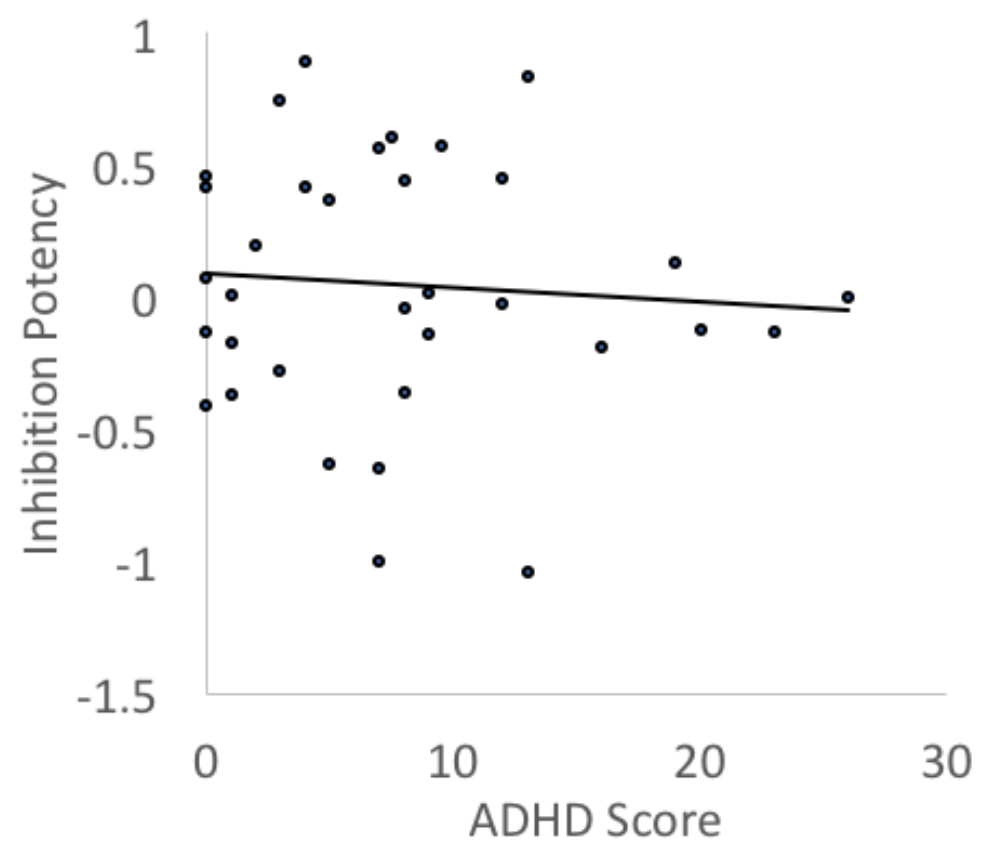

Supplementary FIG. 1. The figure displays a small, non-significant correlation between Inhibition Potency [(sum tics free ticcing - sum suppression) / sum tics free ticcing)] and severity of attention deficit hyperactivity disorder (ADHD) symptoms as measured by the ADHD self-rating scale (ADHD-SB). 


\section{References}

1. Himle MB, Woods DW. An experimental evaluation of tic suppression and the tic rebound effect. Behav Res Ther 2005;43:1443-1451.

2. Sukhodolsky DG, Woods DW, Piacentini J, et al. Moderators and predictors of response to behavior therapy for tics in Tourette syndrome. Neurology 2017;88:10291036.

3. Sambrani T, Jakubovski E, Muller-Vahl KR. New Insights into Clinical Characteristics of Gilles de la Tourette Syndrome: Findings in 1032 Patients from a Single German Center. Front Neurosci 2016;10:415.

4. Muller-Vahl KR, Riemann L, Bokemeyer S. Tourette patients' misbelief of a tic rebound is due to overall difficulties in reliable tic rating. J Psychosom Res 2014;76:472476.

5. Ganos C, Kahl U, Schunke O, et al. Are premonitory urges a prerequisite of tic inhibition in Gilles de la Tourette syndrome? J Neurol Neurosurg Psychiatry 2012;83:975-978.

6. Rosler M, Retz W, Retz-Junginger P, et al. [Tools for the diagnosis of attentiondeficit/hyperactivity disorder in adults. Self-rating behaviour questionnaire and diagnostic checklist]. Nervenarzt 2004;75:888-895.

7. Owens JS, Goldfine ME, Evangelista NM, Hoza B, Kaiser NM. A critical review of self-perceptions and the positive illusory bias in children with ADHD. Clin Child Fam Psychol Rev 2007;10:335-351. 
Author roles:

Valerie Brandt: 1A, 1B, 1C, 2A, 2B, 3A

Julia Kerner auch Koerner: 2C, 3A, 3B

Katja Herrmann: 1C, 2B, 3B

Alexander Munchau: 1A, 3B

1. Research project:
A. Conception,
B. Organization,
C. Execution;

2. Statistical Analysis:
A. Design,
B. Execution,

C. Review and Critique;

3. Manuscript Preparation:
A. Writing of the first draft,
B. Review and Critique 
Financial disclosures for the past 12 months:

Valerie Brandt: has received funding from the Deutsche Forschungsgemeinschaft grant DFG MU 1692/4-1.

Julia Kerner auch Koerner: none.

Katja Herrmann: none.

Alexander Munchau:

Commercial research support by Pharm Allergan, Ipsen, Merz Pharmaceuticals, Actelion;

Honoraria for lectures from Pharm Allergan, Ipsen, Merz Pharmaceuticals, Actelion; GlaxoSmithKline, Desitin and Teva.

Support from the Possehl-Stiftung, Lübeck; the Margot und Jürgen Wessel Stiftung, Lübeck;

Tourette Syndrome Association (Germany); European Huntington Disease Network;

Grants: Multicentre Tics in Children Studies (EMTICS) as part of the FP 7 program

(HEALTH.2011.2.2.1-3); Deutsche Forschungsgemeinschaft (DFG): projects 1692/3-1, 4-1, SFB 936; DFG MU 1692/4-1; Bundesministerium für Bildung und Forschung (BMBF):

DysTract consortium. Innovationsausschuss of the Gemeinsamer Bundesausschuss: Translate NAMSE (structural support for the Lübeck Center for Rare Diseases);

Royalties from the publication of the book Neurogenetics (Oxford University Press) 